\title{
HIV in breastmilk spikes at weaning
}

Findings bolster case for maternal drug therapy beyond feeding's end.

\section{Meredith Wadman}

17 April 2013

The amount of HIV in an infected mother's breast milk spikes when weaning begins, according to a study published today in Science Translational Medicine ${ }^{1}$. The findings are likely to add urgency to efforts to ensure that infected mothers without access to formula take antiretroviral drugs throughout and beyond the time that they wean their infants.

The research also helps to explain why infants who are weaned early and abruptly are no more likely to avoid contracting HIV than do those who continue to breastfeed, a finding from in a randomized clinical trial of 958 HIV-infected women in Lusaka, Zambia, recruited between 2001 and $2004^{2}$.

Grace Aldrovandi, a paediatrician at the Saban Research Institute at the Children's

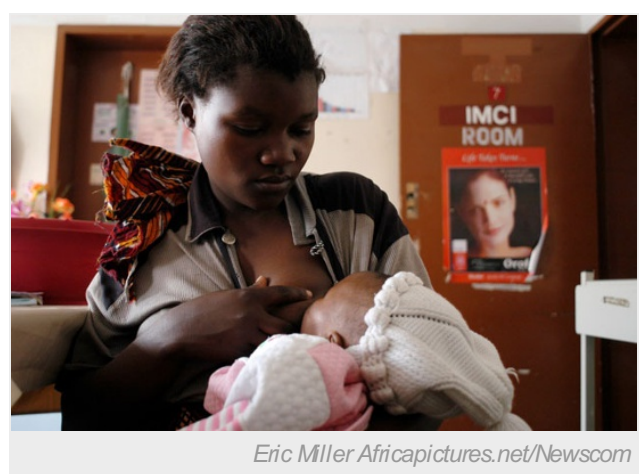

Abrupt weaning raises the amount of HIV in an infected mother's breast milk. Hospital Los Angeles in California, and her colleagues collected milk by pump from all the women in the study after they had exclusively breastfed their infants for four months. The milk from women who then stopped breastfeeding abruptly contained markedly higher levels of HIV than did milk from the women who continued to breastfeed exclusively: a median of 2,708 copies of viral RNA per millilitre of milk compared with fewer than 50 copies per millilitre. In mothers who continued to breastfeed, but not exclusively, the viral levels fell in between those of the two other groups, a median of 293 copies per millilitre.

The differences between the groups remained after researchers adjusted for possible confounders, including how much HIV was in the mother's blood. The team also analysed changes within individual women, and found that in the abrupt weaners, HIV levels in milk rose markedly between samples taken just before weaning and those taken two weeks later; the same was not true at these time points for women who continued to breastfeed.

"Weaning leads to increases in HIV concentrations in breast milk. That's the big message of the paper," says Aldrovandi. "This periweaning period can be quite dangerous."

\section{Dangerous time}

The results have important practical implications, adds Lynne Mofenson, head of the Maternal and Pediatric Infectious Disease Branch at the National Institute of Child Health and Human Development in Bethesda, Maryland. She says it is critical that antiretroviral therapy be given to the mother or infant for the entire duration of breastfeeding, throughout weaning, if mother-to-child transmission of HIV is to be prevented.

The current practice of giving mothers one to two weeks of anti-retroviral therapy after weaning may not be enough, the authors say, given that weaning is a stop-and-start, often protracted, process in real life.

The study also "speaks strongly in favour of exclusive breastfeeding", says Catherine Hankins, deputy director of science at the Amsterdam Institute for Global Health and Development. She notes that viral breast-milk levels were substantially higher in mothers who started weaning their infants in the two weeks before the milk collection than in those who breastfed exclusively.

The authors hypothesize that HIV crosses more easily from blood to milk during weaning because the tight junctions between mammary epithelial cells become leaky, a phenomenon that has been seen various rodents and people ${ }^{3-5}$. Inflammation brought on by abrupt weaning may also have a role, they write. But whatever the biological basis, they say, antiretrovirals will be vital for protecting infants during this time. They suggest follow-up studies to see whether intensified therapy at the time of weaning helps to reduce transmission. 
1. Kuhn, L. et al. Sci. Transl. Med. 5, 181ra51 (2013).

2. Kuhn, L. et al. N. Engl. J. Med. 359, 130-141 (2008).

3. Neville, M. C. et al. Am. J. Clin. Nutr. 54, 81-92 (1991).

4. Nguyen, D. A. \& Neville, M. C. J. Mammary Gland Biol. Neoplasia 3, 233-246 (1998).

5. Nguyen, D. A. et al. J. Endocrinol 170, 347-356 (2001). 